\title{
Interacting two-fluid viscous dark energy models in a non-flat universe
}

\begin{abstract}
We study the evolution of the dark energy parameter within the scope of a spatially non-flat and isotropic Friedmann-Robertson-Walker model filled with barotropic fluid and bulk viscous stresses. We have obtained cosmological solutions that do not have a Big Rip singularity, and concluded that in both non-interacting and interacting cases the non-flat open Universe crosses the phantom region. We find that during the evolution of the Universe, the equation of state for dark energy $\omega \mathrm{D}$ changes from $\omega$ eff $\mathrm{D}>-1$ to $\omega$ eff $\mathrm{D}<-1$, which is consistent with recent observations.
\end{abstract}

Keyword: Cosmology; Dark energy; Theory; Viscous fluid. 